\title{
Le développement de la recherche hydrologique en France en relation avec l'influence de l'homme sur le cycle de l'eau et la prévision des crues*
}

\author{
The development of hydrological research in France relative to \\ the influence of man on water cycles and the forecasting of floods
}

\author{
J. Jacquet
}

Le 16 janvier dernier s'est éteint à Budapest un hydrologue de talent, grand ami de la France, le Docteur Woldemar Laszloffy, qui ne manquait jamais de rappeler que l'organisation des Services hydrauliques de la Hongrie était l'œuvre d'ingénieurs français. En effet, à la suite de la crue catastrophique de la Tisza en 1879, qui détruisit pratiquement la ville de Szeged, une commission internationale avait été commise pour organiser l'annonce des crues de ce fleuve et, sur la proposition des experts français, fut adopté un système dérivé de celui mis en place sur la Seine par Belgrand. Il en fut de même en matière d'irrigation lors de la création - toujours en 1879 - du Bureau national d'hydraulique agricole hongrois : c'est en s'inspirant des ouvrages français sur le drainage et l'irrigation que son $1^{\text {er }}$ directeur E. de Kvassay (qui devait occuper ce poste pendant 40 ans !) élabora ses normes de fonctionnement. En 1901, date de la parution du $1^{\mathrm{er}}$ Manuel d'hydrologie en hongrois, son auteur pouvait écrire dans la préface : " J'ai utilisé surtout des travaux français, étant donné que les bases de l'hydrologie ont été jetées en France, et les études les plus remarquables sont d'origine française. C'est pourquoi, en présentant les lois de l'hydrologie, je cite, dans la plupart des cas, des exemples français..." ".(1)

Ce rappel de l'histoire incite à poser la question : aujourd'hui pourrait-on souscrire à de tels propos ?

Pour ce qui concerne la Hongrie, je crois que la question ne se pose plus, car actuellement ce pays dispose sans doute de l'organisation la plus exemplaire au monde en matière de gestion des eaux, tant du point de vue de la recherche que des services opérationnels. Fait signifi-

(*) Le présent article est extrait du discours de réception à l'Académie d'Agriculture de France de M. Joseph Jacquet, élu membre titulaire dans la Section des sciences physico-chimiques de cette brillante Assemblée. Les discours prononcés à l'occasion de la réception de $M$. Jacquet sont publiés dans le numéro 4 de l'année 1984 des Comptes rendus des séances de l'Académie d'Agriculture de France, 18, rue de Bellechasse, 75007 Paris. catif à noter: ces structures et les hommes qui les animaient ont réussi à traverser sans dommages les multiples bouleversements politiques qu'a connu ce Pays au cours des 70 dernières années, car cette continuité est inscrite dans la nature des choses. En effet, la Hongrie est en quelque sorte une Hollande continentale: l'homme doit combattre chaque année pour reconquérir l'espace cultivable sur les eaux. Dans ses limites actuelles, ce pays est constitué essentiellement d'un fond de cuvette peu perméable, traversé par 2 grands fleuves corsetés de digues dont il faut surveiller sans relâche les apports aux frontières autrichienne et tchèque. Par ailleurs, le régime des précipitations est tel que la majeure part tombe sous forme de neige : lors de la fonte de printemps, si l'on draine trop rapidement l'eau de fusion, les réserves du sol seront insuffisantes pour assurer la production végétale et si l'on laisse s'opérer la percolation pour reconstituer les réserves souterraines, sa lenteur conduit à des durées de submersion des terres qui se traduisent en dommages aux emblavures. L'optimum est donc plus étroit et ne peut se réaliser qu'à partir de prévisions dont la fiabilité soit garantie. Depuis des décennies on pratique en Hongrie une économie de l'eau exemplaire, soutenue par une recherche de qualité dont le rayonnement international de l'Institut des recherches hydrauliques VITUKI est la preuve, tout comme l'exportation des hydrologues hongrois que l'on rencontre maintenant fréquemment en Afrique et ailleurs.

\section{Quelques traits du développement de l'hydrologie en France}

En regard de cette situation, la France fait figure de privilégiée de la nature. Certes on connaît des paroxysmes hydrologiques : la sécheresse de 1976 est encore dans les 
mémoires, l'année 1983 a été ponctuée de nombreuses crues. Mais ces évènements ont une dimension historique, ils sont "millésimés" et la répartition des ressources en eau est en moyenne assez bonne tant dans l'espace que dans le temps. Ceci explique le caractère " en dents de scie " de l'intérêt accordé aux problèmes de l'eau dans notre pays. Avant la prise de conscience des problèmes de pollution, les fluctuations de la ressource ne bénéficiaient pas au départ d'une attention permanente correspondant à une donnée de nature, sauf peut-être de la part d'esprits prospectifs tels Pierre Massé qui, dès 1940, réfléchissant sur la variabilité des apports dans les réservoirs qu'il construisait, jetait les bases de sa stratégie de gestion optimale des réserves hydrauliques en régulant un flux aléatoire par un stock.

Je n'entends pas vous présenter un historique de l'hydrologie en France : c'est un travail qui reste à faire et qui serait certainement d'une réelle utilité pour aider à la définition de structures stables, cohérentes avec des objectifs à long terme bien identifiés pour la recherche hydrologique.

Je noterai cependant deux temps forts du passé :

- la part française dans la naissance de l'hydrologie moderne est incontestable puisque dès 1674 Pierre Perrault a introduit la notion de bassin versant, intégrateur de la pluie, et dressé le premier bilan hydrologique "entréessorties" de la Seine à Aignay-le-Duc. Ce faisant, il amorçait la prise en considération de la dimension "surfacique " ou volumique des phénomènes hydrologiques et non plus la simple dimension "filaire" de ceux de l'hydraulique fluviale [2];

- la $2^{\mathrm{e}}$ moitié du XIX ${ }^{\mathrm{e}}$ siècle a été une période d'intense activité. Un exemple typique est celui de la prévision et de l'annonce des crues de la Loire. C'est à la suite de 2 crues catastrophiques survenues en 1846 et 1856 sur l'ensemble du bassin que fut organisé et mis en place par Comoy un système opérationnel dont l'efficacité reçut une consécration remarquable lors de la grande crue de 1866, dont l'annonce fonctionna correctement [3]. Comptant sur l'illusoire régularité de la série ainsi commencée, on attendit la crue de $1876 \ldots$ On l'attend encore ! Et corrélativement l'attention accordée au système d'annonce et aux travaux de protection s'émoussa avec le temps puisque rien ne se passait... Il fallut attendre les années 1930-1934 pour voir un ingénieur, $M$. Bachet, reprendre le problème et le rénover en mettant au point un système de calcul graphique (les réglettes Bachet) des cotes prévues qui est encore en usage actuellement...

L'histoire récente du développement de l'hydrologie en France a été commandée par des attitudes très pragmatiques, liées à l'extraordinaire dispersion des services de l'Administration et des organismes publics ou parapublics s'intéressant aux problèmes de l'eau. Ces problèmes sont traités en fonction des besoins en mettant en place des cellules de recherches et des structures opérationnelles dont le développement, la stabilisation ou l'extinction éventuelle sont commandés par les impératifs de politique de l'organisme en question. L'exemple d'EDF est significatif à cet égard.

La situation a heureusement évolué, grâce notamment à la loi du 16 décembre 1964 , avec la mise en place de structures opérationnelles qui, après plus de 15 ans de fonctionnement, ont fait la preuve de leur efficacité en matière de gestion des eaux et de pôles de concertation entre Administration et usagers: je veux parler des Agences de bassin et des organes qui les entourent.

\section{La situation de l'hydrologie dans la recherche scientifique française}

En ce qui concerne la recherche hydrologique, la situation est plus floue : je vais tenter d'en dresser un constat à grands traits et de discerner les indices qui paraissent prometteurs de renouveau ou d'avenir.

Précisons d'abord l'objet de cette recherche et les problèmes spécifiques qu'elle pose. L'hydrologie traite des transferts quantitatifs et qualitatifs entre les divers compartiments ou "réservoirs " du cycle des eaux continentales, que ces transferts opèrent par processus mécaniques (précipitation, infiltration, ruissellement, évaporation, écoulement de surface ou souterrain) ou physico-chimiques et biologiques (par interaction eau-sol-plante-atmosphère, par transferts de matière et d'énergie à l'intérieur des écosystèmes aquatiques). La connaissance de la dynamique de ces processus conditionne celle de la formation et du renouvellement de la ressource " eau ", tant en quantité qu'en qualité. Or, plus que jamais, il importe de disposer et donc d'élaborer des outils scientifiques performants permettant de prévoir, à diverses échelles d'espace et échéances de temps, le renouvellement de cette ressource et de contrôler l'influence de l'homme sur les différents compartiments du cycle hydrologique.

Cette approche, d'abord faite au niveau quantitatif des volumes ou débits mis en jeu, doit maintenant aller de pair avec la prise en compte des paramètres de qualité d'eau pour la gestion de la ressource en fonction des utilisations de l'eau et en vue de la protection des écosystèmes aquatiques. L'élaboration de "modèles " vise non seulement la représentation des processus "naturels " d'évolution et de renouvellement des stocks d'eau qui jalonnent le cycle hydrologique d'un bassin mais aussi - et peut-être surtout - l'identification et l'évaluation des perturbations introduites par l'homme tant dans les transferts entre "réservoirs" naturels ou artificiels que dans les facteurs de qualité de l'eau.

Il est donc nécessaire ici de dépasser le stade descriptif des phénomènes et d'accéder à des synthèses de connaissances explicatives des mécanismes physiques, physicochimiques et biologiques qui interviennent tout au long du cycle hydrologique et confèrent à la ressource ses propriétés qualitatives. Le but recherché est de tirer de ces synthèses, des représentations déterministes ou stochastiques des phénomènes de base permettant de simuler avec vraisemblance le cycle de l'eau, dans sa dynamique quantitative et qualitative, à une échelle d'espace donnée (celle du bassin versant ou du lac ou du réservoir) et à des échelles de temps allant de quelques heures à quelques jours (orages et crues), à quelques mois (étiages, sécheresses) ou à une longue période pluriannuelle (pour la gestion des réservoirs et des nappes souterraines) : ces outils de simulation et de prévision n'ont d'autre destination que la gestion de la ressource " eau ", visant la 
satisfaction des besoins et la prévention des nuisances affectant les écosystèmes aquatiques.

II apparaît donc clairement que l'hydrologie est multidisciplinaire par nature et, de ce fait, n'a pu trouver place dans le découpage sectoriel de la recherche française qui reste imprégné de la classification rigide d'Auguste Comte. Elle est ainsi écartelée entre différentes sections du Comité national du CNRS et il n'existe pas à ce jour de formation, rattachée organiquement au CNRS, traitant l'ensemble du cycle de l'eau tel qu'il a été décrit.

C'est pourquoi, les recherches en hydrologie au CNRS sont assez peu nombreuses: elles sont réalisées dans quelques laboratoires associés dépendant du secteur des Sciences de l'Ingénieur (Institut de mécanique de Grenoble, Institut de mécanique des fluides de Toulouse). Récemment encore l'hydrologie était considérée comme une discipline relevant des sciences de la terre et s'apparentant à la géologie $\left({ }^{*}\right)$, et ceci contribuait à entretenir une confusion sur sa nature réelle, notamment avec l'ancienne vision à dominante "descriptive " telle qu'elle était et est encore pratiquée dans certains Instituts de géographie.

Pour ce qui est des Universités, le groupe de recherche le plus important est constitué par le Laboratoire d'hydrologie mathématique de l'Université des sciences et techniques du Languedoc. Mais, faute d'une association avec le CNRS, le renouvellement de cette équipe est fortement handicapé : elle subsiste essentiellement grâce à une politique de contrats avec l'extérieur.

Autre conséquence, sur le plan de la formation cette fois : il n'existe pas d'enseignement spécialisé, spécifique de formation à la recherche en hydrologie. Ceci est vrai aussi pour celle des ingénieurs et techniciens hydrologues, bien qu'il faille cependant noter cette année le démarrage d'une filière "Ressources en eau et aménagement " à l'Ecole nationale supérieure d'hydraulique de Grenoble. $\mathrm{La}$ formation des personnels s'opère soit au sein des organismes qui les recrutent (ORSTOM), soit plus généralement au sein des équipes elles-mêmes... Cette carence est particulièrement ressentie pour le recrutement de jeunes chercheurs.

Dans ces conditions, il n'est pas étonnant que les recherches en hydrologie se soient bien davantage développées dans des organismes relevant de nombreux ministères, dont celui de l'Agriculture: écoles d'ingénieurs, centres de recherches, sociétés d'aménagement, etc.

- en ce qui concerne les écoles d'ingénieurs, il convient de citer :

- l'antenne de l'Ecole nationale du génie rural, des eaux et des forêts à Montpellier, qui travaille en collaboration étroite avec le Laboratoire d'hydrologie mathématique,

- le Centre d'informatique géologique de l'Ecole des mines à Fontainebleau,

- le Centre de recherches sur la gestion des ressources naturelles et de l'environnement (CERGRENE) dépen-

(*) Le Laboratoire d'hydrologie mathématique de l'Université des sciences et techniques du Languedoc à Montpellier a une filiation avec l'Institut de géologie, puis d'hydrogéologie, de cette université. De même il est symptomatique de constater que le Laboratoire de l'Ecole des mines, qui traite des problèmes d'hydrologie souterraine couplée avec l'hydrologie de surface, s'intitule toujours Centre d'informatique géologique... dant de l'Ecole nationale des ponts et chaussées, nouvelle équipe dynamique, mais dont il est encore nécessaire d'asseoir la pérennité;

- pour ce qui est des organismes de recherches, je mentionnerai les équipes de l'ORSTOM, du BRGM, d'EDF, du Bureau de l'eau de la Météorologie nationale, avec les dominantes d'intérêt qui leur sont propres**. Mais je voudrais souligner ici particulièrement la place occupée par des organismes liés directement à l'agriculture :

* I'INRA, avec ses équipes de bioclimatologie et de science du sol, pour la modélisation des échanges eau-sol-plante-atmosphère à diverses échelles de temps et leur intégration jusqu'à l'échelle régionale,

- le CEMAGREF, avec notamment son Département " Ressources en eau " qui constitue aujourd'hui l'une des principales forces organisées en France traitant de l'hydrologie dans sa globalité quantitative et qualitative au sein de ses 2 Divisions : "Hydrologie-Hydraulique fluviale et souterraine ", et "Qualité des eaux - Pêche et pisciculture ". A cette occasion, je tiens à rendre hommage à la clairvoyance des responsables de l'hydraulique du Ministère de l'Agriculture qui, voici une vingtaine d'années, ont constitué ces équipes en leur assignant une mission qu'elles remplissent admirablement. Je n'en veux pour preuve que la qualité des publications qu'elles diffusent et notamment les Cahiers d'informations techniques [4] qui sont des modèles du genre pour faire passer des messages adaptés aux besoins des services opérationnels ou des utilisateurs. Je voudrais enfin souligner l'efficacité de leur collaboration sur le terrain avec les Services régionaux d'aménagement des eaux qui a permis d'intégrer les petits bassins versants d'étude aux réseaux hydrométriques:

- des organismes d'aménagement et de gestion des eaux :

- Sociétés d'économie mixte (je pense au Canal de Provence, aux Côteaux de Gascogne, etc.),

-Agences financières de bassin : elles ne sont pas "acteur" dans la recherche mais "promoteur" d'études générales dans des laboratoires extérieurs par une politique de contrats.

- bureaux d'études parapublics (tels le BCEOM) et privés (SOGREAH par exemple).

Au terme de ce tour d'horizon, on peut ressentir une impression d'éclatement des thèmes de recherches, de dispersion irréductible et finalement de gaspillage de moyens. En fait, ce constat doit être nuancé de remarques importantes pour l'avenir:

- les équipes existantes sont pratiquement toutes de qualité. La compétence des hommes n'est pas en cause et les travaux réalisés soutiennent bien la comparaison avec ce qui se fait à l'étranger;

- la liaisen entre hydrologues appartenant aux différents organismes s'opère efficacement par le truchement de Sociétés savantes, au premier rang desquelles vient la

(**) Pour ne citer qu'un exemple de la qualité des recherches réalisées par ces équipes, on se reportera à la référence [13] de la bibliographie où l'on trouvera un exposé très complet des beaux travaux effectués sous la direction de P. Guillot, au sein du service Ressources en eau de la Division technique générale d'Electricité de France, sur la prédétermination des valeurs extrêmes de crues par la méthode du gradex, méthode maintenant largement utilisée par les projecteurs d'ouvrages. 
Société hydrotechnique de France, d'Associations internationales (Union géodésique et géophysique internationale, Association internationale des sciences hydrologiques, Association internationale des recherches hydrauliques, etc.). A défaut de liens institutionnels, les hommes communiquent quand même !

- il existe dans ce pays des jeunes de valeur attirés par la recherche hydrologique. Le problème est celui de leur recrutement : dans quel cadre ?

- il importe donc de fédérer ces équipes autour d'une politique (qui fait défaut) assurant la continuité de l'effort, l'évaluation de la recherche et le renouvellement des chercheurs.

Il est certain que cette dispersion a entraîné des effets néfastes, notamment des difficultés d'approche globale des problèmes, engendrant parfois des erreurs de décision ou de gestion, faute des connaissances ou d'outils appropriés. Les pouvoirs publics sont conscients du problème : en décembre 1981 le Ministre de l'Environnement a confié à 2 personnalités une mission d'évaluation et de proposition sur les structures et les moyens publics d'étude et de recherche dans le domaine de l'eau. A ce jour, les conclusions de cette mission ne se sont pas encore traduites en décisions.

\section{Que peut-on espérer ?}

Une triple action serait souhaitable pour valoriser ce qui existe :

- sur le plan de la recherche scientifique : une reconnaissance de l'hydrologie comme discipline scientifique dans sa globalité;

- sur le plan de l'administration: une structure de coordination des actions dans ce domaine;

- sur le plan de la formation : une formation spécifique à l'hydrologie tant pour les spécialistes hydrologues que pour les techniciens.

Je voudrais ici mentionner comme un fait nouveau (bien qu'il remonte à 1979) le lancement du Programme interdisciplinaire de recherche sur l'environnement (PIREN) du CNRS, conçu comme antidote à la rigidité des structures sectorielles de la Maison. Ce programme comporte un volet EAU, dont nous allons parler dans un instant, qui a immédiatement attiré des équipes du CNRS très pluridisciplinaires, autres que celles que nous venons de mentionner. Des actions ont été lancées qui commencent à porter des fruits originaux [5].

D'autres faits sont à noter :

- Cette année, pour la $1^{\text {ere }}$ fois, est affiché un poste d'hydrologue comme tel à l'entrée au CNRS;

- le Directeur Général du CNRS demande que soit établi un programme de recherche EAU/CNRS

Il se passe donc quelque chose qui devrait conduire à "démarginaliser" l'hydrologie dans la recherche scientifique.

En attendani, des travaux de qualité se poursuivent et leurs résultats laissent augurer de la valeur ajoutée que les actions proposées pourraient créer. Le bref coup d'œil donné aux 2 thèmes de recherches suivants suffit pour s'en convaincre.

\section{L'influence de l'homme sur le cycle de l'eau}

Je me bornerai à décrire ici les grandes lignes du programme "Hydrologie-gestion de l'eau " du PIREN, en privilégiant les aspects liés à l'utilisation agricole des terres.

Le caractère commun à toutes les actions engagées dans ce cadre est une très forte intégration multidisciplinaire visant toujours l'élaboration d'outils de gestion gagés sur une connaissance des mécanismes qui font évoluer le milieu hydrique d'une situation à une autre.

Trois actions ont été lancées dès 1979 sur les Bassins du Rhône, du Rhin (plaine d'Alsace) et de la Garonne.

- le programme Rhône, à dominante cartographie, est focalisé sur les interactions entre le cours d'eau et sa plaine alluviale qui constitue l'hydrosystème. On a centré l'étude sur la partie du Haut-Rhône non encore aménagée par la Compagnie nationale du Rhône à l'amont de Lyon. L'approche choisie consiste à définir une méthodologie d'établissement de cartes polythématiques synthétisant toute l'information disponible pour la gestion écologique de l'hydrosystème, à partir d'une typologie de la dynamique de ce système fluvial. Dans cette optique, on considère que l'état du réseau fluvial est la résultante d'une histoire qu'il faut prendre en compte pour évaluer les conséquences écologiques des grands aménagements. On est ainsi amené à substituer, à un point "zéro " d'un état "naturel " abstrait, la notion d'état de référence donnant ainsi une dimension historique à la géographie dynamique. L'axe fluvial est considéré en tant que point de convergence des apports d'eau et l'on s'intéresse aux mécanismes de submersion pour des crues d'importances diverses et à l'impact de la durée des submersions sur les écosystèmes. Une partie substantielle des travaux est consacrée aux interventions humaines et à la dynamique de l'environnement (on a réussi à cartographier les interventions humaines depuis 2 siècles dans la plaine du Rhône inondable !), à l'appropriation et à l'utilisation des milieux humides (occupations du sol par les cultures et écoulement des eaux) etc. [6];

- le programme Rhin (ou Alsace) est à dominante "modélisation ", centré sur les problèmes spécifiques à la plaine d'Alsace, c'est-à-dire les interactions eau de surface-solnappe phréatique en relation avec la pollution saline. On s'est attaché à élucider les mécanismes d'alimentation de la nappe phréatique, ceux des champs d'inondation et les échanges nappe-rivière pour différents états des eaux. Ces travaux ont fait l'objet de modélisations, notamment celle des transferts de pollution dans les réservoirs souterrains et par interaction eau-sol-plante. L'ensemble de ces études, complétées par l'évaluation de l'impact des polluants sur l'environnement et la santé (définition de bio-indicateurs), correspond à une approche analytique pour une gestion globale des ressources en eau [7]; 
- le programme Garonne est centré sur l'évaluation de l'impact des pratiques culturales sur les cours d'eau de ce bassin, les indicateurs choisis étant les teneurs de l'eau en phosphore et azote. On y étudie les processus d'évolution de paramètres de qualité d'eau en réponse aux changements d'activités humaines sur les bassins contrôlés, les effets sur l'eutrophisation des secteurs de cours d'eau aménagés (réservoirs), les transferts de pollution azotée dans les eaux souterraines, les modalités de transfert de l'azote dans le sol avec les processus de dénitrification. Des tests de stratégie de régulation de la pollution azotée à l'échelle d'un bassin versant y sont effectués avec analyse des coûts économiques [8].

A ces trois programmes sont venus s'adjoindre d'autres actions à dominante géochimique pour comprendre le fonctionnement d'un bassin en vue de la modélisation de ses exportations de phosphore et d'azote dans des conditions naturelles et perturbées [9].

Un groupe de 3 petits bassins expérimentaux $(0,20$ à $0,75 \mathrm{~km}^{2}$ ) a fonctionné au Mont Lozère depuis 1979. Ses objectifs scientifiques visent les bilans de matière " entrées-sorties " en fonction du couvert végétal et la mise en évidence de différences de fonctionnement hydrologique et géochimique de ces 3 écosystèmes. Il s'agit d'un " laboratoire de terrain " où pourront être étudiés in situ les effets d'interventions humaines sur le couvert végétal [10].

Il apparait donc clairement que tous ces programmes sont fortement structurés sur la mise en évidence et la quantification de l'influence de l'homme sur le cycle de l'eau et les cycles biogéochimiques d'éléments à l'échelle de bassins de diverses tailles. Ils complètent les travaux du CEMAGREF (sur le drainage) [11] et de l'INRA (sur le remembrement), avec une approche plus globale incluant la dimension écologique des impacts. Mais il faut souligner ici que, dans une telle approche, la plus grande rigueur doit présider à la définition des stratégies de mesures à mettre en œuvre en fonction des modélisations à réaliser.

\section{La prévision des crues}

Problème ancien s'il en est, mais toujours d'actualité : après chaque crue, qui s'est traduite par des submersions importantes et des pertes matérielles, on ne manque pas de s'interroger sur les conditions d'avertissement des riverains de l'occurence et de l'importance du phénomène [12].

Pourquoi ?

Je ferai simplement ici quelques remarques :

- la distribution des crues dans le temps est parfaitement aléatoire et, tant qu'il ne se produit pas de phénomènes à répétition, personne ne soulève la question;

- les systèmes de prévision sur les grands bassins fluviaux Seine, Rhône, Loire, Garonne sont techniquement valables, mais il importe de les réviser en permanence pour tenir compte des non stationnarités des conditions d'écoulement liées aux aménagements ou modifications du lit. On dispose maintenant de techniques bien au point pour corriger efficacement ces dérives [13];

- de nouveaux besoins de prévision apparaissent, notamment sur les bassins amont où il faut développer d'autres types de modèles de relations pluie-débit. Il en existe un arsenal varié, mais en général le problème sur lequel on bute est celui de la disponibilité de données adéquates pour le calage du modèle choisi qu'il faudra souvent dégrader en le simplifiant pour le rendre cohérent avec l'information expérimentale dont on dispose [14];

- pour ces bassins amont, une meilleure utilisation de prévisions quantifiées de précipitations par des Services d'annonces de crues décentralisés est souhaitable;

- la télédétection de la pluie par radar et le suivi du déplacement du corps pluvieux devront être de plus en plus intégrés dans l'information "pluie reçue par le bassin ", en complément (et non en substitution) du réseau de pluviographes classique. Le problème de l'estimation de la lame d'eau reçue par le bassin n'est pas toujours résolu de façon satisfaisante [15];

- les progrès techniques les plus importants concernent les réseaux de télémesure et systèmes d'acquisition et de traitement de données au centre de prévision : on dispose là encore d'une palette étendue de systèmes éprouvés;

- la méthodologie d'élaboration de modèles de prévision avec les stratégies de mesures associées est bien au point; - la cartographie des zones inondables en fonction de la fréquence des crues peut être envisagée, car on dispose maintenant de guides "normalisés " d'estimation des probabilités des débits de crues [16] [17];

- par contre, la méthodologie d'estimation des dommages d'inondations est à revoir.

La prévision hydrologique se heurte toujours à 2 difficultés : d'une part une insuffisance ou inadéquation d'observations pour ajuster convenablement le modèle de calcul, d'autre part l'apparition de "dérives dans le temps " de ses paramètres dues à la non stationnarité des conditions locales. Pour remédier à ce dernier défaut, on " asservit" le modèle en le "recalant " en permanence, en fonction des écarts constatés entre prévision et observation, par un dispositif de commande optimale, tel que le filtre de Kalman bien connu des automaticiens. Cette technique a fait ses preuves en hydrologie et l'utilisation de telles méthodes " adaptatives " pour la prévision des crues est maintenant devenue courante [18].

Une question fondamentale reste posée : Y-a-t-il augmentation du nombre et de l'importance des crues dues au changement de régime des eaux par l'action de l'homme?

- nous ne parlerons pas ici des crues en zone urbanisée qui constituent un problème en soi, sur lequel d'importantes recherches sont en cours, notamment au sein du CERGRENE et du Laboratoire d'hydrologie mathématique de Montpellier;

- pour ce qui concerne le nombre et la distribution dans le temps des crues fluviales, on peut répondre par la négative, à l'échelle historique tout au moins (quelques siècles) : les séries sont stationnaires. Mais la caisse de résonance des médias joue à son avantage pour donner une impression d'amplification du phénomène...;

- on constate des modifications d'hydrogrammes de crues à l'exutoire de parcelles drainées ou en terroir rural. 
Il importe d'être prudent dans l'intégration de ces résultats à l'échelle d'un bassin étendu. Les observations du changement en hydrologie nécessiteront encore de nombreuses mises au point méthodologiques (cf. Programme PIREN/EAU);

- les pratiques agricoles, drainage et assainissement, ont des effets parfois antagonistes dont il est malaisé de prévoir la résultante sur les crues;

- la mise en culture des zones inondables (en remplacement des prairies et peupleraies) fait problème surtout poùr l'accroissement des dommages.

Quelques autres problèmes peuvent être évoqués :

- l'incidence des manœuvres coordonnées d'ouvrages sur l'évolution de la crue;

- l'utilisation des zones inondables pour atténuer les pointes de crues.

Le problème majeur concernant les crues reste celui de la qualité de l'information du public. La diffusion des résultats des recherches dans ce domaine est affaire de Pénélope... Un dernier exemple l'illustre :

Fin avril 1983 se produisit une crue de l'Allier qui provoqua d'importantes submersions de terres agricoles de la Basse Limagne occasionnant, en raison de sa date tardive, des dégats importants aux terres déjà traitées en prévision des semailles du maïs semence.

La presse locale couvrit l'événement et donna de très bonnes informations sur la genèse et l'évolution du phénomène, qui n'avait rien d'extraordinaire du point de vue de sa puissance. Le journal titrait " La crue de l'Allier : grave parce que... hors saison ". Jusqu'ici rien à dire, mais dans un encadré on pouvait lire ceci :

Cette crue de l'Allier mérite tout juste d'être notée sur les tablettes des riverains. En effet avec un maximum de $3,90 \mathrm{~m}$ à Coudes, elle se situe presque en queue de classement (...), ce qui n'est rien si l'on sait que pour celle centenaire de 1866 il y avait (...) $8,90 \mathrm{~m}$ d'eau sous (et sur) le vieux pont de Coudes (...)

$$
9 \text { mètres en } 1988 \text { ? }
$$

Précision intéressante, ce genre de phénomène est cyclique. Et l'on peut d'ores et déjà prévoir qu'en 1988-90 - ce n'est pas si loin - l'Allier un jour mettra la barre à près de 9 mètres. Et l'urbanisation a bien changé depuis 1866 . Les villages ne sont plus seulement sur les collines. Ils se sont créés dans la plaine...

On se trouve ici en présence d'une désinformation caractérisée. Je n'en rends pas responsable le journaliste, encore qu'il eût du faire préciser à son informateur (sans doute au Service d'annonces des crues) ce que signifie " crue centenaire", dont la durée de retour est 100 ans, et dont l'occurrence a lieu en moyenne 1 fois par siècle. Mais il peut y en avoir soit $0,1,2$ ou plus sur une période de 100 ans donnée! On peut d'ailleurs calculer les probabilités correspondantes :

Probabilité 1 crue centenaire en 100 ans $=0,63$

d'avoir 2 crue centenaire en 100 ans $=0,26$

au moins 3 crue centenaire en 100 ans $=0,08$

Cet exemple montre de plus qu'il faut constamment démystifier la recherche des périodicités en hydrologie. Il est certain que, si de tels cycles existaient, la prévision des phénomènes en serait sérieusement facilitée. Les chercheurs de " cycles " tentent d'en découvrir en analysant par filtrage (moyenne mobile ou moyenne mobile pondérée) un échantillon de données portant sur une longue période : ils perdent leur temps car Slutzky a démontré que l'application de ces procédés à des séries chronologiques tirées "au hasard" introduisait des périodicités fictives. Si l'analyse statistique n'est pas guidée par des relations causales connues par ailleurs, elle ne peut être un instrument de découverte... [19].

\section{Conclusion}

En guise de conclusion, permettez-moi d'insister sur un point qui conditionne l'avenir et le développement de la recherche hydrologique au service d'une politique de l'eau : celui des réseaux de mesure permanents.

Toute politique de protection, d'aménagement des eaux, de gestion de la ressource en eau, pour laquelle quantité et qualité sont indissociables, vise le bien commun et le long terme. Sa mise en œuvre requiert de la part des pouvoirs publics une continuité d'action sous-tendue par un effort de recherche pluridisciplinaire à la mesure des enjeux techniques, économiques et sociaux concernés. Cette continuité passe par l'organisation rationnelle et l'exploitation de réseaux de mesure permanents, permettant de suivre l'évolution d'indicateurs d'état des milieux aquatiques constitutifs de notre cadre de vie. Il s'agit là d'une tâche prioritaire entre toutes, d'un instrument indispensable au progrès des connaissances. Sa mise en œuvre comporte deux volets : l'un en matière de développement d'instrumentation spécifique qualifiée pour l'équipement de ces réseaux, l'autre est une revalorisation de la fonction de concepteur et de gestionnaire de ces réseaux de mesure permanents, dont les données permettent seules de juger, à terme, de l'efficacité de la politique réalisée pour la protection ou la régénération de ressources en eau de qualité. Cette tâche permanente de recueil d'informations hydrologiques est éminemment du ressort de l'Administration, en tant que garante du bien commun : il ne peut s'agir que d'une action persévérante et obstinée, dépassant les turbulences de la conjoncture.

\section{Références bibliographiques}

[1] LASZLOFFY W., 1977. - Influences françaises sur le développement des Services hydrauliques de la Hongrie. Mémoires de l'Académie des Sciences. Inscriptions et Belles Lettres de Toulouse, vol. 139, $15^{e}$ série, t. VIII, 106-126.

[2] DOOGE J.C.I., 1959. - Un bilan hydrologique au xvile siècle. La Houille Blanche, $\mathrm{n}^{\circ}$ 6, nov. 1959, 799-803.

[3] COMOY M., c. 1860. - Renseignements sur les crues de la Loire et de ses grands affluents. Règles pratiques pour déterminer à l'avance sur différents points du littoral la hauteur et l'époque du maximum des crues de la Loire. Impr. Montalon, Chalon-sur-Saône, 32 p., 4 pl. h.t.

[4] Notes d'hydrologie dans les Cahiers d'Informations Techniques du CEMAGREF. 


\section{L'influence de l'homme sur le cycle de l'eau}

[5] 1983. - Images de l'Environnement. Courrier du CNRS. Supplément au $n^{\circ} 52.120$ p. (Programme PIREN).

[6] PIREN, 1982. - Cartographie polythématique appliquée à la gestion écologique des eaux : étude d'un hydrosystème fluvial, le Haut-Rhône français. Editions du CNRS, Centre Régional de Publication, Lyon, 113 p. .+ cartes h.t.

[7] ZILLIOX L., 1982. - L'action de recherche interdisciplinaire du groupe PIREN/EAU en Alsace. Rech. Géogr. Strasbourg, 19/20/21, 241-247.

[8] CAPBLANCQ J. et DAUTA A., 1983. - Relations entre les rivières et leur bassin versant : exemple du système Garonne. Courrier du CNRS, Supplément au n $\mathrm{n}^{\circ} 52,56-61$.

[9] LAUGA J. et DECAMPS H., 1983. - Modeling the effects of Natural Conditions and Land use on the export rate of non-point source pollutants. Com. Coll. Unesco, MAB 5, oct. 1983, Budapest.

[10] DUPRAZ C., LELONG F., TROY J.P., 1982. - Comparative Study of the effects of vegetation on the hydrological and hydrochemical flows in three minor catchments of Mont Lozère (France). Methodological aspects and first results. Acte of Sympos. Hydrol. Research Basins, Berne, sept. 1982, 671-681.

[11] OBERLIN G., 1981. - Influence du drainage et de l'assainissement rural sur l'hydrologie. Bulletin d'Informations du CEMAGREF, $\mathrm{n}^{\circ} 285$, oct. 1981.

\section{La prévision des crues}

[12] Seine Normandie $n^{\circ}$ 48. Automne 1983. - Agence de Bassin Seine Normandie. $35 \mathrm{p}$. ( $\mathrm{n}^{\circ}$ consacré aux crues).
[13] Crues et Précipitations intenses: système d'annonce - prévision - valeurs extrêmes. Institut National Polytechnique de Grenoble. Formation Professionnelle Continue. Stage de formation pour Techniciens, Ingénieurs et Cadres du 31 janvier au 3 février 1983. Ecole Nationale Supérieure d'Hydraulique, 1983.

Fasc. I : Rappels de météo générale.

Fasc. II : Réseaux de mesure et systèmes d'acquisition.

Fasc. III : Prévisions des crues et modèles de la relation pluie-débit.

Fasc. IV : Prédétermination des valeurs extrêmes de crues. (Excellent panorama des résultats de la recherche hydrologique en France dans ce domaine.

[14] Relations pluie-débit. La Houille Blanche, nº́cial 4-5/1980, 219-346.

[15] Radar et précipitations. La Houille Blanche, n 5-6, 1983.

[16] MIQUEL J., 1982. - Guide d'estimation des probabilités de crues. $E D F / D E R / L N H$, Rapport $H E$ 43/82-22, juillet 1982 , 2 parties + Annexes.

[17] BERNIER J. et MIQUEL J., 1979. - Incertitudes de l'information et décisions en situation de risque. Cas de problèmes de protection contre les crues. La Houille Blanche, $\mathrm{n}^{\circ} 4-5$, $1979,251-258$.

[18] MIQUEL J., 1982. - Filtre de Kalman et annonces de crues : La Loire à Tours, La Saône à Couzon. EDF/DER/ LNH. Rapport HE 43/82-12, avril 1982.

[19] BERNIER J., 1965. - Sur les probabilités des sècheresses et des étiages. EDF, Bull. du Centre de Recherche et d'Essais de Chatou, n 11, avril 1965, 3-12. (Sur la recherche des périodicités en hydrologie et l'effet Slutzky). 


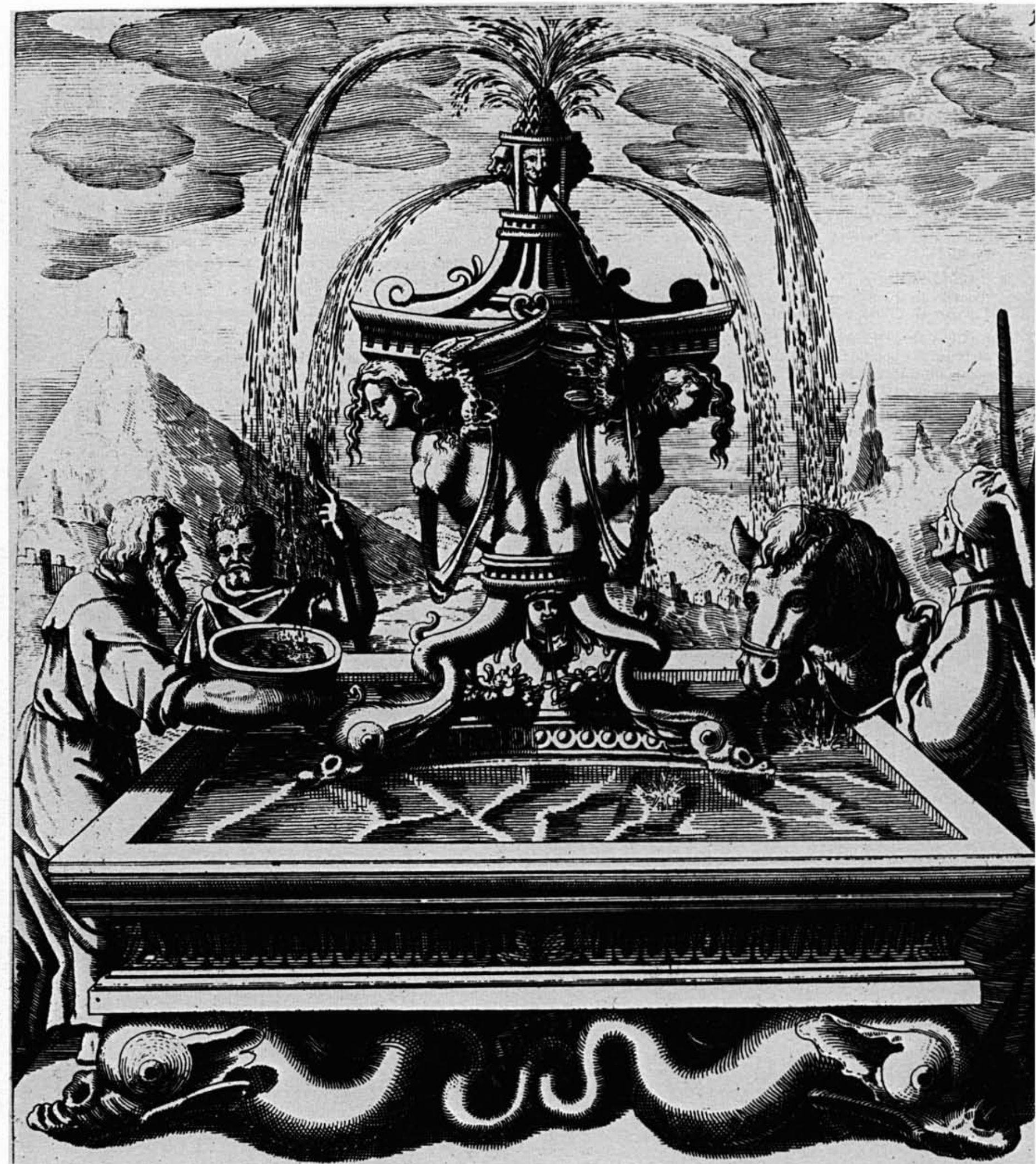

